\title{
Numerical limit analysis of the ultimate load bearing capacity of a pier foundation under complex load
}

\author{
$H F$ Wang $^{*}$, and $X H$ Cheng \\ Department of Civil Engineering, Tsinghua University, Beijing 100084, China
}

\begin{abstract}
A pier foundation has plenty of advantages as the foundation form for large onshore wind turbines in the terrain of the Gobi desert and collapsible loess areas. The ultimate load bearing capacity design, as an important part in the design phase of this foundation form, is not sufficiently in terms of design theory and the checking method, especially for application in collapsible loess areas. In this paper, numerical limit analysis has been employed to analyze the load bearing capacity for the scheme of selection of a pier foundation of a $100 \mathrm{MW}$ wind farm in certain collapsible loess geology in Ningxia. The results were then compared with the empirical formulas, the limit equilibrium solutions and the finite element solutions to verify the accuracy of the results. It has been demonstrated that under the ultimate engineering load, the pier foundation can meet the stability requirement. In the ultimate state, whether the surrounding soil of the foundation falls in plasticity is associated with the ratio of the depth to the diameter of the foundation. The numerical limit analysis method can automatically determine the failure surface.
\end{abstract}

\section{Introduction}

Due to advantages like rich resources, a good economic effect and low pollution, wind power has gained extensive attention worldwide.

According to the forces acting on it, the conventional foundation of a wind turbine includes a gravity type foundation, a pier foundation and a pile foundation. The gravity type foundation counteracts the external forces and bending moments acting on the wind turbine using the weight of the concrete and backfill and the corresponding couples. A pile foundation can be further classified as an ordinary reinforced concrete pile and rock micropile foundation, relying on the weight of the foundation's platform together with the pile soil to counteract the external loads. These two foundation forms are common and have been widely applied with relatively complete design methods. As shown in Fig.1, the pier foundation originated in America [1,2], with a general burial depth of $8 \mathrm{~m}$ to $11 \mathrm{~m}$ and a top width of the foundation between $5 \mathrm{~m}$ and $9 \mathrm{~m}$. The overall geometry is an inverted cylinder, relying mainly on the force couple caused by the interaction of the soil pressures of the in-situ soil and the foundation's concrete to counteract the external loads.

\footnotetext{
*Corresponding author: timwang517@tsinghua.edu.cn
} 
Large-scale development of onshore wind power has occurred relatively late in China, and imported technology has played the main role in its development. In regions with good soil properties like Xinjiang, the pier foundation has broad applications. However, as for the collapsible loess areas, the pier foundation should be applied with caution due to the mechanical mechanisms and limitations in the calculation methods.

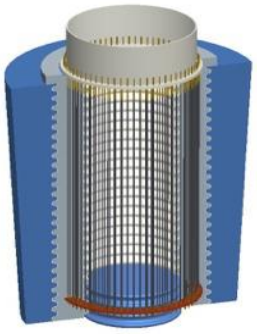

Fig. 1. Typical situation of a pier foundation for a wind turbine.

At present, the analysis methods for the ultimate anti-overturning load bearing capacity of a pier foundation mainly include the limit equilibrium method [3, 4], the plastic upper limit analysis [5, 6] and the finite element method [7-10]. Generally, the limit equilibrium method considers the foundation and the soil mass inside it as a rigid entity and performs a series of approximation and assumptions relating to key parameters such as soil pressure distribution and depth of the center of rotation. Engineering experience indicates that this method can reasonably estimate the foundation's stability, but the accuracy cannot be guaranteed theoretically. The plastic upper limit analysis employs the kinematically admissible velocity field to satisfy the constraints, including the strain compatibility, the velocity boundary conditions and the associative flow rules, and the theoretical basis is strict. Nevertheless, the analytical capability of this method is limited since it is difficult to perform load bearing capacity analysis for complex foundations or load conditions. The finite element method has distinct advantages in dealing with complex problems, which is one of the major methods used for the performance analysis of a pier foundation. However, in the design phase, an error range between the finite element calculation value and the actual engineering load bearing capacity is not foreseeable, and it is difficult to properly select the constitutive model and parameters relating to the soil mass for specific engineering projects.

Based upon the conventional limit analysis theorem, the numerical limit analysis is a numerical method that utilizes finite element discretization technology to discretize the soil mass and the structure, it establishes the kinematically admissible velocity field (upper limit) and the static admissible stress field (lower limit), and applies a planning algorithm to obtain the maximum (upper limit) and minimum (lower limit) ultimate load. This method has the following advantages: (1) without assumption of the failure mode, it automatically acquires the failure mechanism, the plastic zone of the soil and the stress distribution through program optimization; (2) it has the capability of dealing with complex loads and complex geometric conditions with the finite element method, and has a strict theoretical basis for limit analysis; (3) it envelops the true results by analysis of the upper and lower limits and is capable of providing the error range.

Numerical element limit analysis concept was proposed by Lysmer in 1970 [11]. Sloan introduced the steepest edge active set algorithm in 1988, so that the efficiency of the algorithm was greatly improved [12]. Sloan [13], as well as Sloan and Kleeman [14], calculated the undrained vertical load bearing capacity of a strip foundation by applying upper limit and lower limit analyses of the finite element limit, respectively. Ukritchon et al. $[15,16]$ calculated the undrained ultimate load bearing capacity and stability of a foundation pit support for a strip foundation under complex force conditions by applying 
the numerical limit analysis. Theoretical study and the engineering application of numerical limit analysis started late in China. Yang Feng et al. [17] analyzed the stability of a shallow tunnel by upper limit analysis of the numerical limit. Huang et al. [18, 19], as well as Li and Wang [20] calculated the load bearing capacity and stability of a side slope for a strip foundation by using the lower limit analysis of the finite element limit, respectively.

The correct treatment of the contact relationship (tangential and normal phases) on the bucket-soil interface is one of the key factors in the accuracy of the simulation. The friction strength of the bucket-soil interface is associated with the material properties of the bucket and the soil, which can be measured using the model test. In the present work, the friction strength of the bucket-soil interface and the undrained shear strength were assumed to be different.

Nowadays, researchers at home and abroad have developed three-dimensional numerical limit analysis technology $[19,21]$, but the analyses are limited when dealing with simple problems like a strip foundation and a homogeneous soil layer due to limited processing capacity. A relatively mature two-dimensional plane strain program can analyze more complicated problems with relatively illustrative results. Some previous investigations have proved that there is a slight difference between the load bearing capacity of a complex load of a shallow round foundation acquired from three-dimensional analysis, and that acquired from a plane strain analysis [10]. Therefore, the work in this paper employed the plane strain numerical limit analysis.

\section{A brief introduction to the principles of numerical limit analysis}

The principles of numerical limit analysis fall into two parts: the finite element discretization method and the limit analysis solution. A brief introduction to the principles of both the lower limit analysis and the upper limit analysis has been given below.

The simplified analysis model for calculating the ultimate load bearing capacity of a pier foundation by using numerical limit analysis has been shown in figure 2(a). The top of the foundation was aligned with the ground, and the foundation was in the soil. The center of the top of the bucket was subject to gravity (V), the horizontal force $(\mathrm{H})$ and the overturning moment (M). A rigid-plastic assumption was used for the soil. Since the analysis mainly considered the interaction between the soil and the structure rather than the bending of the steel structure itself, the foundation was assumed to be rigid, that is, 1) a single structural element is rigid without internal strain; 2) the adjacent structural elements share the same angle of rotation and displacement at the same node coordinate without considering a plastic hinge.

\subsection{Principles of the lower limit analysis}

In the lower limit analysis, the soil was discretized as 3-node constant-strain-rate triangle elements. As shown in figure 2(a), each node had three stress components, and each stress component was assumed to be linear within the element. This was different from the conventional finite element, in finite element limit analysis each node belongs and only belongs to one element (i.e., multiple nodes may share the same coordinates), so that the stress discontinuity is caused at the boundary of the adjacent elements. A suction caisson was discretized as 2-node beam elements and a single-node plastic hinge element (without real dimensions); each node belonged to one element and had three degrees of freedom (two acting forces and one bending moment). It was supposed that the stress was linearly distributed along the structural elements. The configuration of the plastic hinge unit in the 
upper and lower limit analyses was there to simulate a rigid-plastic structure. As the foundation was assumed to be rigid, no plastic hinge did arise.

In the lower limit analysis, the static admissible stress field was established, and it could be taken as a linear planning model to solve the objective function (see table 1). The constitutive relationship of soil used the Tresca yield criterion. Since a linear planning algorithm as used, the linearization treatment of the yield function as performed using the inscribed regular 24-gon (lower limit) and the circumscribed regular 24-gon (upper limit) (figure 2(c)). For the detailed formulas and analysis procedures, refer to Sloan [13].

The objective function of the lower limit analysis is the maximum external load that satisfies the conditions of the static admissible stress field. Regarding the pier foundation, the maximum external load was from the transverse force and the overturning torque was transferred from the upside of the wind turbine and gantry, and both resulted from the effect of the wind on the structures.

\subsection{Principles of the upper limit analysis}

In the upper limit analysis, the discretization forms of the soil and structure were the same as those in the lower limit analysis (figure 2(b)). Each node of the soil element and beam element had two velocity components, and the node of the plastic element had three velocity components. The distribution of the velocity field was linear in element. Each node independently belonged to one element, and thus a velocity discontinuity was allowed at the boundary of the adjacent elements.
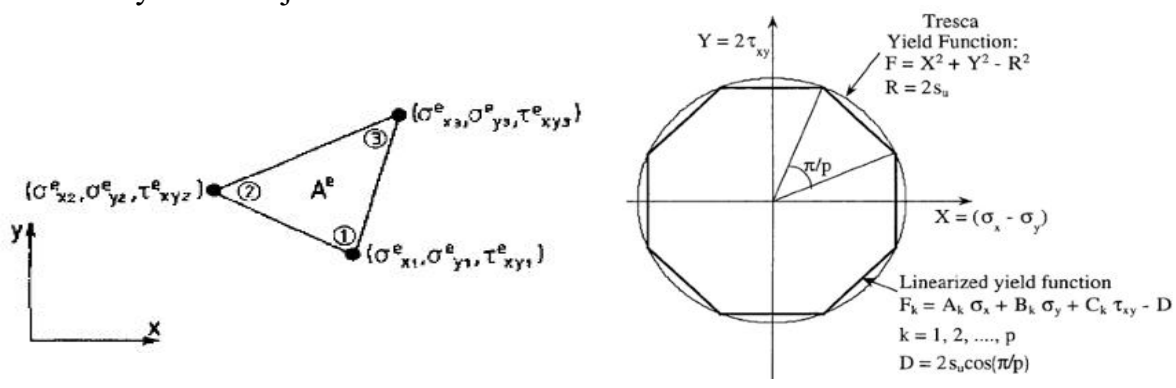

(a)

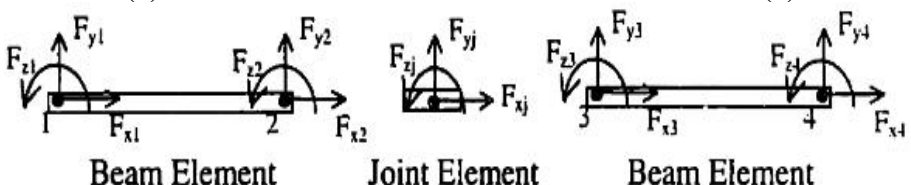

(b)

Fig. 2. Problem discretization for caisson foundation and summary of plain-strain elements in numerical limit analyses: (a) Lower bound discretization for soil elements, (b) Lower bound discretization for structural elements, (c) Tresca yield criterion for lower bound analyses.

In the upper limit analysis, a kinematically admissible velocity field was established, and a minimal external force meeting the requirements was obtained after the planning (see table 1). For detailed formulas and procedures, refer to Sloan and Kleeman [14].

The objective function of the upper limit analysis is obtained by equalizing the work of the external and internal forces. Especially for the problems analyzed in this paper, the external forces could be classified as: (1) weight of the soil and the bucket, (2) horizontal load and overturning torque at the top of the bucket; and the internal forces could be 
classified as: (1) plastic deformation inside the soil element and between elements, (2) slip and cracks at the soil-structure interface.

Table 1. Solution of the numerical limit analyses.

\begin{tabular}{|c|c|c|}
\hline Limit analyses & Lower & Upper \\
\hline Objective function & $\operatorname{MAX} .\left\{\mathbf{C}_{1}^{\mathrm{T}} \sigma\right\}$ & MIN. $\left\{\mathbf{C}_{2}{ }^{\mathrm{T}} \dot{\lambda}+\mathbf{C}_{3}{ }^{\mathrm{T}} \mathbf{U}^{ \pm}\right\}$ \\
\hline \multirow{4}{*}{ Constraint function } & {$\left[\begin{array}{cc}\frac{\partial}{\partial x} & \frac{\partial}{\partial y} \\
\frac{\partial}{\partial y} & \frac{\partial}{\partial x}\end{array}\right]\left[\begin{array}{ll}\sigma_{x} & \tau_{x y} \\
\sigma_{y} & \tau_{x y}\end{array}\right]=\left[\begin{array}{l}0 \\
\gamma\end{array}\right]$} & $\begin{array}{l}\dot{\varepsilon}_{x}=-\frac{\partial u}{\partial x}=\dot{\lambda} \frac{\partial F}{\partial \sigma_{x}}, \dot{\varepsilon}_{y}=-\frac{\partial v}{\partial y}=\dot{\lambda} \frac{\partial F}{\partial \sigma_{y}} \\
\dot{\varepsilon}_{x y}=-\left(\frac{\partial v}{\partial x}+\frac{\partial u}{\partial y}\right)=\dot{\lambda} \frac{\partial F}{\partial \tau_{x y}}, \dot{\lambda} \geq 0 \\
F=X^{2}+Y^{2}-R^{2}=0\end{array}$ \\
\hline & $\sigma_{n 1}=\sigma_{n 2}, \tau_{1}=\tau_{2}$ & $\begin{array}{l}\Delta u_{\mathrm{n}}=\left|\Delta u_{\mathrm{t}}\right| \tan \phi,\left|\Delta u_{\mathrm{t}}\right|=u_{\mathrm{t}}^{+}+u_{\mathrm{t}}^{-} \\
\Delta u_{\mathrm{t}}=u_{\mathrm{t}}^{+}-u_{\mathrm{t}}^{-}, u_{\mathrm{t}}^{+} \geq 0, u_{\mathrm{t}}^{-} \geq 0\end{array}$ \\
\hline & $F=X^{2}+Y^{2}-R^{2} \leq 0$ & $u_{1}=d_{1}, v_{1}=p_{1}$ \\
\hline & $\sigma_{n 1}=q_{1}, \tau_{1}=t_{1}$ & $W_{\mathrm{ext}}=\int_{L} \sigma_{\mathrm{n}} v_{\mathrm{n}} d L+\int_{A} \gamma \nu d A=W_{\mathrm{int}}$ \\
\hline
\end{tabular}

\section{Engineering example}

\subsection{Overview of the engineering and numerical simulation}

A wind farm has been proposed for the Ningxia Hui Autonomous Region, China, with a scale of $100 \mathrm{MW}$. The stratum is loess-like soil (Q41). The loess-like silt is soil with medium-high compressibility, and it is characterized by its collapsibility, and is classified as Class-I (slight) non-weight collapsible loess to Class-II (medium) self-weight collapsible loess. The stratum is continuously distributed in the field with a thickness of $0.5-10.5 \mathrm{~m}$. The main physical and mechanical indicators of each specific layer of rock and soil have been listed in table 2 .

For the foundation of a wind turbine, the vertical load is its own weight, and the horizontal load and the overturning torque is caused by the forces from the wind acting on the structure. As provided by the supplier of the wind turbine, the weight of the wind turbine and the associated structure was $3,891 \mathrm{kN}$, and the ultimate horizontal load and the ultimate overturning load at the bottom of the structure were $854 \mathrm{kN}$ and 71,413 kN.m, respectively (combined and corrected according to the norm [22]).

Table 2. Parameters of the soil strata.

\begin{tabular}{|c|c|c|c|c|c|}
\hline \multirow{2}{*}{$\begin{array}{c}\text { Name of soil } \\
\text { layer }\end{array}$} & $\begin{array}{c}\text { Coefficient of } \\
\text { compressibility } \\
a_{1 \sim 2}\end{array}$ & $\begin{array}{c}\text { Volume } \\
\text { weight } \\
\mathrm{g} /\left(\mathrm{kN} \cdot \mathrm{m}^{-3}\right)\end{array}$ & $\begin{array}{c}\text { Cohesion } \\
c_{\mathrm{q}} / \mathrm{kPa}\end{array}$ & $\begin{array}{c}\text { Saturated quick shear test } \\
\text { friction } \\
j /\left(^{\circ}\right)\end{array}$ & $\begin{array}{c}\text { Modulus of } \\
\text { compressibility } \\
/ \mathrm{MPa}\end{array}$ \\
\hline Loess-like silt -1 & 0.39 & 15.3 & 7 & 24.1 & 5.04 \\
\hline Loess-like silt -2 & 0.218 & 17.7 & 8.4 & 21.7 & 8 \\
\hline
\end{tabular}


This paper has proposed a standard selection of the type of foundation and has conducted three transformation modes for different foundation dimensions according to other methods, with consideration of the requirements of penetrating the wet limit layer, so as to analyze the effects of increasing the diameter and rim grout on the foundation's load bearing capacity. One of the main advantages of numerical limit analysis is that a true value can be enveloped within the upper and lower limit solutions.

Model-1: $D_{1}$ (upside diameter) $=8 \mathrm{~m}, D_{2}$ (lower diameter $)=7 \mathrm{~m}, D$ (mean diameter $)=$ $7.45 \mathrm{~m}, L$ (foundation depth) $=11 \mathrm{~m}$;

Model-2: the mean diameter of the foundation $D$ was extended by $1 \mathrm{~m}, 1.5 \mathrm{~m}$ and $2 \mathrm{~m}$ in the same direction (i.e., increasing the $\mathrm{C} 20$ grouting range outside), and the corresponding mean outer diameters of the bucket were $8.45 \mathrm{~m}, 8.95 \mathrm{~m}$ and $9.45 \mathrm{~m}$.

Model-3: kept the upper diameter of the bucket $D_{1}$ and the outer side slope constant, and the burial depth of the foundation was increased to $11 \mathrm{~m}, 12 \mathrm{~m}$ and $13 \mathrm{~m}$, respectively.

Model-4: the C20 grouting range was locally extended in the upper part of the bucket, an extension was conducted at a burial depth of $4 \mathrm{~m}$ and the extension's diameter was $11 \mathrm{~m}$, and then it was gradually extended to a diameter of $12 \mathrm{~m}$ at the top.

\subsection{Factor of safety and method validation}

Factor of safety $(F S)$ is the ratio of the foundation's ultimate load bearing capacity to its ultimate load under certain conditions of its own weight and $M / H$. All models $F S$ results are shown in table 3 . Considering the compatibility of safety and economy, the follow-up scheme used the data results of Model-1. Therefore, a FS check was performed on Model-1 using the soil stratification, the method of limit equilibrium method, the coefficient of subgrade reaction and the ultimate bearing capacity method in table 4 .

Table 3. Factors of safety of the pier foundations.

\begin{tabular}{|c|c|c|c|c|c|c|c|c|}
\hline \multirow{2}{*}{$\begin{array}{c}\text { Model } \\
\text { types }\end{array}$} & \multirow{2}{*}{ Model-1 } & \multicolumn{3}{|c|}{ Model -2 } & \multicolumn{3}{c|}{ Model -3 } & \multirow{2}{*}{ Model -4 } \\
\cline { 3 - 8 } & & $D=8.45$ & $D=8.95$ & $D=9.45$ & $L=11$ & $L=12$ & $L=13$ & \\
\hline$F S$ & 1.31 & 1.46 & 1.64 & 1.82 & 1.24 & 1.44 & 1.68 & 1.65 \\
\hline
\end{tabular}

Table 4. Comparison of the different method's FS for Model-1.

\begin{tabular}{|c|c|c|c|}
\hline Validating method & $\begin{array}{c}\text { Soil stratification } \\
\text { method }\end{array}$ & $\begin{array}{c}\text { Coefficient of subgrade } \\
\text { reaction }\end{array}$ & $\begin{array}{c}\text { Ultimate bearing } \\
\text { capacity method }\end{array}$ \\
\hline$F S$ & 1.37 & 1.29 & 1.40 \\
\hline
\end{tabular}

\subsection{Failure mechanism}

The failure mechanisms (i.e., the deformation failure modes) and the soil plastic zone distribution that were solved by the upper limit solution of the finite element limit analysis have been shown in figure 3 and figure 4 . Obviously, under all load conditions, the basic failure mechanism was dominated by rotation around a point in the foundation. In all cases, the soil around the bucket would be destroyed. However, in Model-4, the proportion of the damaged area would be large. In fact, the assumption of the rigidity of the soil in the bucket was used in the case of $L / D>2$ at first. As to the foundation of an onshore wind turbine, since the main effect comes from the overturning moment rather than from the horizontal force or the uplift force, the value of $L / D$ is considerably reduced and less than 2 in general, and can be close to 1 especially in Model-4. In this case, the assumption of the rigidity of the soil in the bucket was no longer true. Nonlinear finite element analysis requires optimization of the constitutive equations to reflect the soil plasticity caused by the rotation of the principal stress so as to improve the estimation accuracy of the plastic zone. 

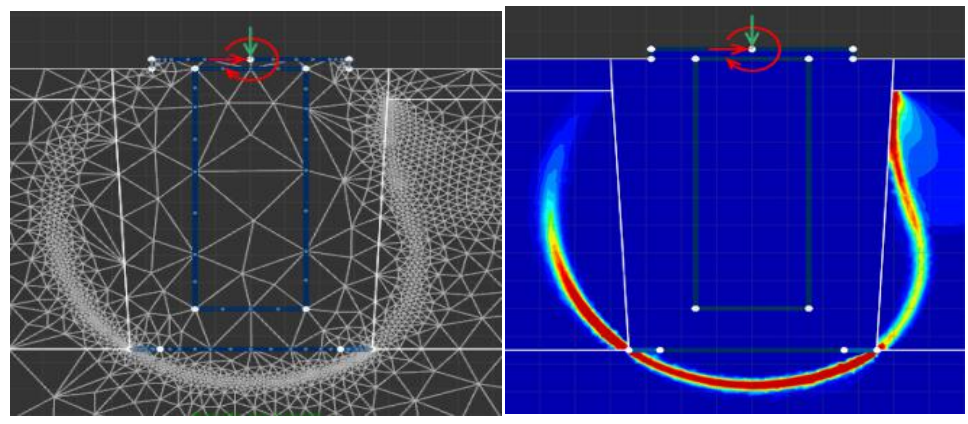

Fig. 3. Contours of the numerical limit analyse.

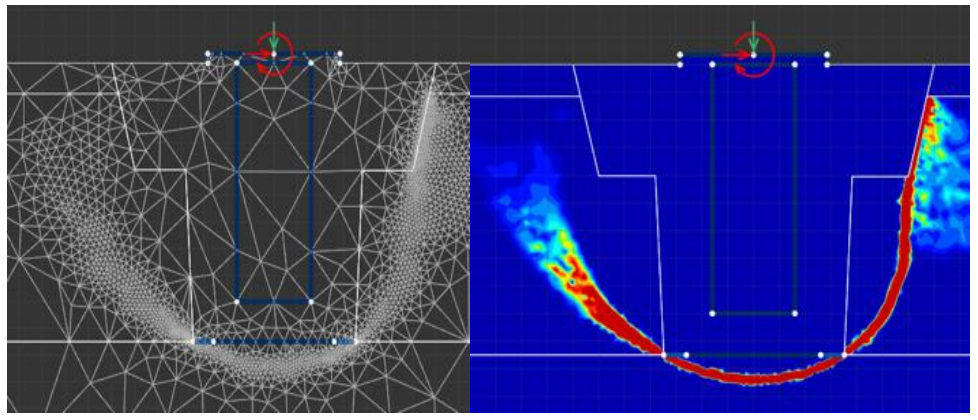

Fig. 4. Contours of the numerical limit analyses for Model-4.

\section{Conclusions and suggestions}

This paper has used numerical limit analysis to study the ultimate load bearing capacity of the pier foundation of a 2-MW wind turbine in a wind farm in Ningxia under the combined effect of gravity, horizontal loads and overturning moments. Assumption of the failure modes was not needed in the analysis, and the basic failure mechanisms, the soil plastic zone and the soil stress distribution could be automatically obtained through optimization. The main conclusions and suggestions were as follows:

(1) Concerning pier foundation type selection, the factor of safety of the ultimate load bearing capacity for different dimensions have been provided and the factor of safety of the schemes were compared. This indicated that the basic type selection with a diameter of 7.45 $\mathrm{m}$ and a depth of $11 \mathrm{~m}$ was the optimal scheme.

(2) Based on the comparison between the empirical formulas, the limit equilibrium method, the method of the coefficient of subgrade reaction and the finite element solution, it can be seen that the numerical limit analysis was characterized by a strict theorem and the calculation results thereof were reliable and accurate.

(3) With these address parameters, increasing the foundation depth and diameter could dramatically enhance the ultimate load bearing capacity, and the effect of increasing the diameter was superior to that of increasing the foundation depth in increasing the antioverturning load bearing capacity. This requires further analysis to find out whether this conclusion also applies to other soil properties.

(4) Numerical limit analysis could automatically distinguish the development of a crack at the soil-structure interface and the calculation results were more reasonable.

\section{References}

1. Henderson A P and Patrick M B 1996 Tensionless pier foundation EP,US5586417 
2. Henderson A P and Patrick M B 1998 Pier foundation under high unit compression US, US5826387

3. Sun X Y, Luan M T and Tang X W 2010 Study of horizontal bearing capacity of bucket foundation on saturated soft clay ground Rock Soil Mech. 31 667-72

4. Clukey E C and Morrison M J 1993 A centrifuge and analytical study to evaluate suction caissons for TLP applications in the Gulf of Mexico Design and Performance of Deep Foundations: Piles and Piers in Soil and Soft Rock (ASCE) pp 141-56

5. Aubeny C and Murff J D 2005 Simplified limit solutions for the capacity of suction anchors under undrained conditions Ocean Eng. 32 864-77

6. Zhang J H, Chen Z Y and Li F 2010 Three dimensional limit analysis of suction bucket foundations Ocean Eng. 37 790-9

7. Li D Y, Feng L Y, Guo Y X and Cao L X 2013 Numerical analysis of lateral bearing behaviors of skirted suction caissons Chin. J. Geo. Eng. 35 33-8

8. Wang Z Y, Luan M T, Yang Q, Wang Z T and Zhang Q Y 2009 Numerical analysis of ultimated bearing capacity behavior of suction caisson foundations under cyclic loading J. Dalian Univ. Techno. 49 414-8

9. Randolph M and House A 2002 Analysis of suction caisson capacity in clay Offshore Technology Conf.

10. Bransby M F and Yun G J 2009 The undrained capacity of skirted strip foundations under combined loading Geotechnique 59 115-25

11. Lysmer J 1970 Limit analysis of plane problems in soil mechanics Journal of Soil Mechanics \& Foundations Div 96 1311-34

12. Sloan S W 1988 A steepest edge active set algorithm for solving sparse linear programming problems Int. J. Numer. Meth. Eng. 26 2671-85

13. Sloan S W 1988 Lower bound limit analysis using finite elements and linear programming Int. J. Numer. Anal. Met. 12 61-77

14. Sloan S W and Kleeman P W 1995 Upper bound limit analysis using discontinuous velocity fields Comput. Method. Appl. M. 127 293-314

15. Ukritchon B, Whittle A J and Sloan S W 1998 Undrained limit analyses for combined loading of strip footings on clay J. Geotech. Geoenviron. 124 265-76

16. Ukritchon B, Whittle A J and Sloan S W 2003 Undrained stability of braced excavations in clay $J$. Geotech. Geoenviron. 129 738-55

17. Yang F, Yang J S, Zhang X M and Zhao L H 2010 Finite element analysis of upper bound solution of shallow-buried tunnel stability in undrained clay Chin. J. Rock Mech. Eng. 29 3952-59

18. Huang Q W, Huang M S and Wang G H 2007 Calculation of bearing capacity of strip footing using lower bound limit method Chin. J. Geo. Eng. 29 572-9

19. Huang Q W and Jia C Q 2007 3D analysis of ultimate bearing capacity by numerical limit analysis Chin. J. Geo. Eng. 29 1809-14

20. Li Z and Wang J X 2007 Lower bound limit study on plastic limit analysis of rock slope using finite elements based on nonlinear programming Chin. J. Rock Mech. Eng. 26 747-52

21. Salgado R, Lyamin A V, Sloan S W and Yu H S 2004 Two-and three-dimensional bearing capacity of foundations in clay Geotechnique $\mathbf{5 4}$ 297-306

22. China renewable energy engineering institute 2008 FD003-2007 Design regulations on subgrade and foundation for wind turbine generator system (Peking: China Water Power Press) 